MSC 47D03, 47D06, 47D07, 60K25, 60J22

DOI: $10.14529 / \mathbf{m m p} 180303$

\title{
LORD KELVIN AND ANDREY ANDREYEVICH MARKOV IN A QUEUE WITH SINGLE SERVER
}

A. Bobrowski, Lublin University of Technology, Lublin, Poland, a.bobrowski@pollub.pl

We use Lord Kelvin's method of images to show that a certain infinite system of equations with interesting boundary conditions leads to a Markovian dynamics in an $L^{1}$ type space. This system originates from the queuing theory.

Keywords: queue; method of images; generation theorem; boundary conditions; Markovian dynamics.

Dedicated to Professor Jan Kisyński on the occasion of his 85th birthday.

\section{Introduction}

\section{Background}

As shown by an increasing body of examples, an intelligent enrichment of an underlying state space may change the nature of a problem under consideration and allow for its elegant solution. For instance, even the simplest scalar delay-differential equation

$$
x^{\prime}(t)=a x(t)+b x(t-\tau), \quad t \geq 0,
$$

where $a, b \in \mathbb{R}$ and $\tau>0$ are given, does not lead to a well-posed problem if we think of $x(t) \in \mathbb{R}$ as a state of a process at time $t$ and desire to describe dynamics in $\mathbb{R}$. One should rather think of the evolution of "history segments of $x$ ", i.e., say continuous, functions

$$
u_{t}:[-\tau, 0] \rightarrow \mathbb{R}, \quad t \geq 0,
$$

defined by $u_{t}(s)=x(s+t), s \in[-\tau, 0]$ so that $u_{t}$ contains the entire information on $x$ in the time interval $[t-\tau, t]$. Then, (1) may be rewritten as a differential equation in the space $C[-\tau, 0]$ of continuous functions on $[-\tau, 0]$ and the related initial value (Cauchy) problem is well-posed. As it transpires, this rather simple idea leads to a quite satisfying theory and has far-reaching consequences (see [1, Section VI.6] or [2] and references given there).

For another instance, a time-inhomogeneous Markov process $(X(t))_{t \geq 0}$ in a state $S$ becomes time-homogeneous if extended to the pair $(X(t), t)_{t \geq 0}$ and considered in the Cartesian product space $S \times \mathbb{R}^{+}$. Similarly, embedding the state-space of a non-Markovian process in a more suitable space may lead to Markovian dynamics (see e.g. [3]). Hidden Markov models, abounding in machine learning and biological sequence analysis [4,5], are the other side of the same coin. Here, although the process is in fact Markovian from the very beginning, what we observe is only a part or shadow of its state-space.

A similar trick to that described for time-inhomogeneous Markov process allows reducing non-autonomous Cauchy problems to autonomous ones (see [1, Section VI.9, Evolution Semigroups]). However, interestingly, in contrast to the examples presented 
above, this transformation, presumably meant to simplify analysis, rarely helps in practice; in fact, I know of no particular instance were it is really useful.

The next example is at least intriguing. As developed by Samuel Goldstein [6] and Mark Kac [7], the stochastic process

$$
\xi(t)=\int_{0}^{t}(-1)^{N_{a}(s)} \mathrm{d} s, \quad t \geq 0,
$$

where $N_{a}(t), t \geq 0$ is a Poisson process with expected value $\mathbb{E} N_{a}(t)=a t$ ( $a$ is a positive constant), lies behind a probabilistic formula for telegraph equation. In [8] Jan Kisyński proves that the process

$$
\left(\xi(t),(-1)^{N_{a}(t)}\right)
$$

which is $\xi(t)$ "enriched" by the coordinate $(-1)^{N_{a}(t)}$, has independent increments in the non-commutative group $\mathbb{R} \times\{-1,1\}$ with the following multiplication rule:

$$
(\tau, k) \circ(\varsigma, l)=(\tau l+\varsigma, k l) .
$$

In other words, $\xi(t), t \geq 0$ is a Lévy process whereas $\left(\xi(t),(-1)^{N_{a}(t)}\right), t \geq 0$ is a Markov process. While the former information suffices for a successful treatment of the telegraph equation (see e.g. $[9,10]$ ), the latter gives an additional insight and allows for natural generalizations [8].

\section{A Markovian Approach to a Single Server Queue}

Our paper is devoted to an example of similar type originating from the queuing theory. It is well-known that unless quite restrictive conditions are imposed, a process of the form

$$
N(t)=\# \text { of customers in a queue at time } t
$$

is non-Markovian [11]. However, as developed by e.g. D.R. Cox [12], in the case of M/G/1 queue (with Markov-type arrivals, general distribution of service time and one server), the two-dimensional process

$$
(N(t), x(t)), t \geq 0,
$$

where $x(t)$ is the time the customer being served has spent at a service point up to time $t$, is Markov. Recently, the latter idea has been reinvestigated by P. Gwiżdz in [13], where it was noted that the resulting process may be viewed as a piece-wise deterministic process of M.H.A. Davies [14-16] (see also [17]). Gwiżdż's paper contains (see Theorem 2.1 there) a proof of the fact that the system

$$
\begin{aligned}
\frac{\partial p_{0}(t)}{\partial t} & =-\alpha p_{0}(t)+\int_{0}^{\infty} \mu(x) p_{1}(t, x) \mathrm{d} x \\
\frac{\partial p_{1}(t, x)}{\partial t} & =-\frac{\partial p_{1}(t, x)}{\partial x}-(\alpha+\mu(x)) p_{1}(t, x) \\
\frac{\partial p_{n}(t, x)}{\partial t} & =-\frac{\partial p_{n}(t, x)}{\partial x}-(\alpha+\mu(x)) p_{n}(t, x)+\alpha p_{n-1}(t, x), n \geq 2, x>0,
\end{aligned}
$$


(where $\alpha>0$ is intensity of customers' arrivals, and $\mu(\cdot)$, a non-negative, bounded function is the hazard rate function for service time) supplemented with the following boundary conditions:

$$
\begin{aligned}
& p_{1}(t, 0)=\int_{0}^{\infty} p_{2}(t, x) \mu(x) \mathrm{d} x+\alpha p_{0}(t) \\
& p_{n}(t, 0)=\int_{0}^{\infty} p_{n+1}(t, x) \mu(x) \mathrm{d} x, \quad n \geq 2,
\end{aligned}
$$

has, for any initial data, a unique mild solution in a certain space of type $L^{1}$ (see further on for details). Here, $p_{n}(t, x)$ is the probability density that at time $t$ there are $n \geq 1$ customers and the one being served has spent time $x$ at the service point; $p_{0}(t)$ is the probability that at that time there are no customers in the queue. From the perspective of the theory of semigroups of operators, this theorem says that the related operator is the generator of a semigroup in this space. The latter semigroup governs the evolution of probability distributions of the process (2), and the fact that the semigroup is composed of Markov operators is expressed in the relation

$$
p_{0}(t)+\sum_{n=1}^{\infty} \int_{0}^{\infty} p_{n}(t, x) \mathrm{d} x=1,
$$

which holds for all $t>0$ provided it holds for $t=0$.

\section{Our Goal}

Theorem 2.1 in [13] is obtained by a Greiner-like [18] domain-perturbation technique, extended to include unbounded domain-perturbations in $L^{1}$-type spaces, and designed in such a way that the perturbed semigroup remains positive if the original semigroup is positive (original Greiner's perturbation does not posses this feature). In this paper, we will show the same result more directly, using Lord Kelvin's method of images [19-24]. Here is the main idea of the proof.

If all the terms involving $\alpha$ or $\mu$ are removed from system (3)-(5), and boundary conditions (6) are disregarded, the resulting equations may be solved explicitly on the entire real line by the very simple formula:

$$
p_{0}(t)=p_{0}(0), \quad p_{n}(t, x)=p_{n}(0, x-t), \quad t \geq 0, x \in \mathbb{R}, n=1,2, \ldots,
$$

provided $p_{0}(0)$ and $p_{n}(0, x)$ are known for all $x \in \mathbb{R}$. Since restoring all removed terms is a matter of a bounded perturbation, the question of solving (3)-(6) reduces to that of existence of a procedure which, given $\alpha$ and $\mu$, assigns to $p_{n}(0, \cdot), n \geq 1$ defined on $\mathbb{R}^{+}$ their unique extensions to the entire $\mathbb{R}$ in such a way that functions (7), as restricted to $x \geq 0$, solve (3)-(5) (with appropriate terms removed) and, at the same time, boundary conditions (6) are satisfied. Note that although in the latter equations $\alpha$ and $\mu$ may be thought to be set to zero, they remain non-zero in the boundary conditions (6).

To prove that this idea works well is the aim of this paper.

\section{The Main Theorem}

Throughout the paper we assume that $\mu$ is a bounded, non-negative function on $\mathbb{R}^{+}$ and $\alpha>0$ is a positive constant. 
Let $L^{1}\left(\mathbb{R}^{+}\right)$be the space of absolutely continuous functions on $\mathbb{R}^{+}$, and let

$$
L:=\mathbb{R} \times l^{1}\left(L^{1}\left(\mathbb{R}^{+}\right)\right)
$$

be the space of sequences $\left(f_{n}\right)_{n \geq 0}$ where $f_{0}$ is a real number and $f_{n}, n \geq 1$ are members of $L^{1}\left(\mathbb{R}^{+}\right)$, such that

$$
\left\|\left(f_{n}\right)_{n \geq 0}\right\|_{L}:=\left|f_{0}\right|+\sum_{n=1}^{\infty}\left\|f_{n}\right\|_{L^{1}\left(\mathbb{R}^{+}\right)}<\infty
$$

when equipped with this norm, $L$ is a Banach space. In what follows, we will often use the following bounded linear functional on $L$ :

$$
\Sigma_{I}\left(f_{n}\right)_{n \geq 0}=f_{0}+\sum_{n=1}^{\infty} \int_{0}^{\infty} f_{n}(x) \mathrm{d} x .
$$

Let $\mathcal{D}$ be the set of $\left(f_{n}\right)_{n \geq 0} \in L$ such that

- each $f_{n}, n \geq 1$ is absolutely continuous with $f_{n}^{\prime} \in L^{1}\left(\mathbb{R}^{+}\right)$,

- $\sum_{n=1}^{\infty}\left\|f_{n}^{\prime}\right\|_{L^{1}\left(\mathbb{R}^{+}\right)}<\infty$.

Also, let $F_{n}: \mathcal{D} \rightarrow \mathbb{R}$ be the linear functionals given by

$$
\begin{aligned}
& F_{1}\left(f_{m}\right)_{m \geq 0}=f_{1}(0)-\int_{0}^{\infty} \mu(x) f_{2}(x) \mathrm{d} x-\alpha f_{0}, \\
& F_{n}\left(f_{m}\right)_{m \geq 0}=f_{n}(0)-\int_{0}^{\infty} \mu(x) f_{n+1}(x) \mathrm{d} x, \quad n \geq 2 .
\end{aligned}
$$

Here is the main theorem of the paper.

Theorem 1. The operator $A_{\mu, \alpha}$ defined on the domain

$$
\mathcal{D}\left(A_{\mu, \alpha}\right)=\mathcal{D} \cap \bigcap_{n \geq 1} \operatorname{ker} F_{n}
$$

by the formula

$$
A_{\mu, \alpha}\left(f_{n}\right)_{n \geq 0}=\left(g_{n}\right)_{n \geq 0}
$$

where

$$
\begin{aligned}
& g_{0}=-\alpha f_{0}+\int_{0}^{\infty} \mu(x) f_{1}(x) \mathrm{d} x, \\
& g_{1}=-f_{1}^{\prime}-(\alpha+\mu) f_{1}, \\
& g_{n}=-f_{n}^{\prime}-(\alpha+\mu) f_{n}+\alpha f_{n-1}, \quad n \geq 2,
\end{aligned}
$$

generates a strongly continuous semigroup of Markov operators in L.

To recall, a bounded linear operator $P: L \rightarrow L$ is said to be a Markov operator, if $P\left(f_{n}\right)_{n \geq 0}$ is non-negative when $\left(f_{n}\right)_{n \geq 0}$ is, and for such $\left(f_{n}\right)_{n \geq 0}$ we have $\Sigma_{I} P\left(f_{n}\right)_{n \geq 0}=$ $\Sigma_{I}\left(f_{n}\right)_{n \geq 0}$. If the latter equality is replaced by the inequality $\Sigma_{I} P\left(f_{n}\right)_{n \geq 0} \leq \Sigma_{I}\left(f_{n}\right)_{n \geq 0}$ the operator is said to be sub-Markov. 


\section{Reduction to a Simpler Case}

We will show now that Theorem 1 may be deduced from the following result.

Theorem 2. The operator $B_{\mu, \alpha}$ defined on the domain $\mathcal{D}\left(B_{\mu, \alpha}\right)=\mathcal{D}\left(A_{\mu, \alpha}\right)$ by the formula

$$
B_{\mu, \alpha}\left(f_{n}\right)_{n \geq 0}=\left(g_{n}\right)_{n \geq 0},
$$

where

$$
g_{0}=0, \quad g_{n}=-f_{n}^{\prime}, \quad n \geq 1,
$$

generates a strongly continuous semigroup of positive operators in L.

Remark 1. We stress again that, while $\alpha$ and $\mu$ are not featuring in the definition of the "action" of $B_{\mu, \alpha}$, both are involved in the definition of its domain. Hence, what we are facing here is a domain-changing perturbation; such perturbations were studied in detail by G. Greiner [18], and the approach presented in [13] follows Greiner's path. We are using a different method, i.e., Lord Kelvin's method of images that has been developed as a way to deal with boundary conditions in semigroup theory in [19-23] and [24]. For yet different ways of dealing with boundary conditions see [25] and [26].

Suppose thus that $B_{\mu, \alpha}$ generates a semigroup of positive operators and then choose a $\kappa$ such that

$$
\kappa \geq \sup _{x \geq 0} \mu(x) .
$$

Then, for any $\left(f_{n}\right)_{n \geq 0} \in \mathcal{D}\left(A_{\mu, \alpha}\right)=\mathcal{D}\left(B_{\mu, \alpha}\right)$,

$$
A_{\mu, \alpha}\left(f_{n}\right)_{n \geq 0}=B_{\mu, \alpha}\left(f_{n}\right)_{n \geq 0}+C_{\mu, \alpha, \kappa}\left(f_{n}\right)_{n \geq 0}-(\alpha+\kappa)\left(f_{n}\right)_{n \geq 0},
$$

where $C_{\mu, \alpha, \kappa}$ is the bounded, non-negative, linear operator defined by

$$
C_{\mu, \alpha, \kappa}\left(f_{n}\right)_{n \geq 0}=\left(g_{n}\right)_{n \geq 0}
$$

with

$$
\begin{aligned}
& g_{0}=\int_{0}^{\infty} \mu(x) f_{1}(x) \mathrm{d} x+\kappa f_{0}, \quad g_{1}=(\kappa-\mu) f_{1}, \\
& g_{n}=(\kappa-\mu) f_{n}+\alpha f_{n-1}, n \geq 2 .
\end{aligned}
$$

Thus, by the Phillips perturbation theorem, $B_{\mu, \alpha}+C_{\mu, \alpha, \kappa}$ generates a semigroup of positive operators and this implies that so does $A_{\mu, \alpha}$, because the last two operators differ by a constant multiple of the identity operator.

Hence, we are left with showing that the semigroup generated by $A_{\mu, \alpha}$ is composed of Markov operators. A well-known necessary and sufficient condition for that is for $\lambda\left(\lambda-A_{\mu, \alpha}\right)^{-1}$ to be Markov operators for all sufficiently large $\lambda>0$. Since $A_{\mu, \alpha}$ generates a semigroup, for sufficiently large $\lambda$ the resolvent equation

$$
\lambda\left(f_{n}\right)_{n \geq 0}-A_{\mu, \alpha}\left(f_{n}\right)_{n \geq 0}=\left(g_{n}\right)_{n \geq 0}
$$


has a unique solution for all $\left(g_{n}\right)_{n \geq 0}$. Because the semigroup generated by $A_{\mu, \alpha}$ is composed of positive operators, the map assigning solution $\left(f_{n}\right)_{n \geq 0}$ to $\left(g_{n}\right)_{n \geq 0}$ is positive also (since the resolvent is the Laplace transform of the semigroup). Applying the functional $\Sigma_{I}$ to both sides of the resolvent equation we see thus that all we need to prove is that

$$
\Sigma_{I} A_{\mu, \alpha}\left(f_{n}\right)_{n \geq 0}=0
$$

for all non-negative $\left(f_{n}\right)_{n \geq 0} \in \mathcal{D}\left(A_{\mu, \alpha}\right)$.

To this end, we first use (8) to calculate (writing, for simplicity of notation, $\int_{\mathbb{R}^{+}} \mu f_{n}$ instead of $\left.\int_{0}^{\infty} \mu(x) f_{n}(x) \mathrm{d} x\right)$

$$
\sum_{n=1}^{\infty} f_{n}(0)=\alpha f_{0}+\sum_{n=2}^{\infty} \int_{\mathbb{R}^{+}} \mu_{n} f_{n}, \quad\left(f_{n}\right)_{n \geq 0} \in \mathcal{D}\left(A_{\mu, \alpha}\right) .
$$

On the other hand, with similar notation,

$$
\Sigma_{I} A_{\mu, \alpha}\left(f_{n}\right)_{n \geq 0}=-\alpha f_{0}-\sum_{n=2}^{\infty} \int_{\mathbb{R}^{+}} \mu_{n} f_{n}-\sum_{n=1}^{\infty} \int_{\mathbb{R}^{+}} f_{n}^{\prime} .
$$

Since $\int_{\mathbb{R}^{+}} f_{n}^{\prime}=-f_{n}(0)$, these two relations combined imply (9), completing the proof.

Remark 2. The last calculation shows that (9) holds in fact for all vectors $\left(f_{n}\right)_{n \geq 0}$ in $\mathcal{D}\left(A_{\mu, \alpha}\right)$ whether positive or not. Hence, $\lambda\left(\lambda-A_{\mu, \alpha}\right)^{-1}$ preserves the functional $\Sigma_{I}$, i.e., $\Sigma_{I} \lambda\left(\lambda-A_{\mu, \alpha}\right)^{-1}\left(f_{n}\right)_{n \geq 0}=\Sigma_{I}\left(f_{n}\right)_{n \geq 0}$ for all sufficiently large $\lambda$. Therefore, the semigroup generated by $A_{\mu, \alpha}$ also preserves this functional, i.e., the value of $\Sigma_{I}$ does not change along this semigroup's trajectories (this is a stronger condition than that of being composed of Markov operators).

\section{A Semigroup in a "Larger" Space}

A first step in the procedure of the Lord Kelvin method of images is to construct a semigroup generated "by $B_{\mu, \alpha}$ without boundary conditions" in a "larger" space. This is our goal in this section.

Let $\left(f_{n}\right)_{n \geq 0} \in L$, and consider an $n \geq 1$. Any extension of $f_{n}$ to a function $f_{n}^{\diamond}$ on $\mathbb{R}$ is determined by $g_{n}(x)=f_{n}^{\diamond}(-x)$, a function on $\mathbb{R}^{+}$. It would be nice to have $g_{n} \in L^{1}\left(\mathbb{R}^{+}\right)$ but this is rarely the case. Fortunately, for the particular extension we are looking for there is an $\omega \geq 0$ such that all functions $x \mapsto \mathrm{e}^{-\omega x} g_{n}(x)$ are in $L^{1}\left(\mathbb{R}^{+}\right)$. Hence, we introduce $L_{\omega}^{1}\left(\mathbb{R}^{+}\right)$as the space of functions $g: \mathbb{R}^{+} \rightarrow \mathbb{R}$ such that $x \mapsto \mathrm{e}^{-\omega x} g(x)$ is a member of $L^{1}\left(\mathbb{R}^{+}\right)$. When equipped with the norm

$$
\|g\|_{L_{\omega}^{1}\left(\mathbb{R}^{+}\right)}=\int_{0}^{\infty}|g(x)| \mathrm{e}^{-\omega x} \mathrm{~d} x
$$

$L_{\omega}^{1}\left(\mathbb{R}^{+}\right)$is a Banach space. 
A pair $(f, g) \in L^{1}\left(\mathbb{R}^{+}\right) \times L_{\omega}^{1}\left(\mathbb{R}^{+}\right)$may be identified with a function $h$ on $\mathbb{R}$ : it suffices to agree that $h(x)=f(x), x \geq 0$ and $h(x)=g(-x)$ for $x<0$. The space $L_{\omega}^{1}(\mathbb{R})$ of functions $h: \mathbb{R} \rightarrow \mathbb{R}$ such that

$$
\|h\|_{L_{\omega}^{1}(\mathbb{R})}:=\int_{0}^{\infty}|h(x)| \mathrm{d} x+\int_{0}^{\infty} \mathrm{e}^{-\omega x}|h(-x)| \mathrm{d} x
$$

is finite, is a Banach space with this norm, and the spaces $L^{1}\left(\mathbb{R}^{+}\right) \times L_{\omega}^{1}\left(\mathbb{R}^{+}\right)$and $L_{\omega}^{1}(\mathbb{R})$ are isometrically isomorphic. All our extensions will be members of $L_{\omega}^{1}(\mathbb{R})$ and sequences of extensions will be members of

$$
L_{\omega}:=\mathbb{R} \times l^{1}\left(L_{\omega}^{1}(\mathbb{R})\right)
$$

the space of sequences $\left(h_{n}\right)_{n \geq 0}$ where $h_{0}$ is a real number and $h_{n}, n \geq 1$, are members of $L_{\omega}^{1}(\mathbb{R})$, such that

$$
\left\|\left(h_{n}\right)_{n \geq 0}\right\|_{L_{\omega}}:=\left|h_{0}\right|+\sum_{n=1}^{\infty}\left\|h_{n}\right\|_{L_{\omega}^{1}(\mathbb{R})}<\infty ;
$$

when equipped with this norm, $L_{\omega}$ is a Banach space.

The formula

$$
T(t) h(x)=h(x-t), \quad t \geq 0, x \in \mathbb{R},
$$

defines a strongly continuous semigroup in $L_{\omega}^{1}(\mathbb{R})$, and since

$$
\begin{aligned}
\|T(t) h\|_{L_{\omega}^{1}(\mathbb{R})} & \leq \int_{0}^{t}|h(x-t)| \mathrm{d} x+\int_{t}^{\infty}|h(x-t)| \mathrm{d} x+\int_{0}^{\infty}|h(-x-t)| \mathrm{e}^{-\omega x} \mathrm{~d} x \\
& \leq \mathrm{e}^{\omega t} \int_{0}^{t} \mathrm{e}^{-\omega x}|h(-x)| \mathrm{d} x+\int_{0}^{\infty}|h(x)| \mathrm{d} x+\mathrm{e}^{\omega t} \int_{t}^{\infty} \mathrm{e}^{-\omega x}|h(-x)| \mathrm{d} x \\
& \leq \mathrm{e}^{\omega t}\|h\|_{L_{\omega}^{1}(\mathbb{R})},
\end{aligned}
$$

the operator norm of $T(t)$ does not exceed $\mathrm{e}^{\omega t}$. A standard argument shows that the domain $\mathcal{D}(G)$ of the generator $G$ of $\{T(t), t \geq 0\}$ is composed of absolutely continuous members $h$ of $L_{\omega}^{1}(\mathbb{R})$ such that $h^{\prime} \in L_{\omega}^{1}(\mathbb{R})$, and $G h=-h^{\prime}$ on this domain.

It follows that

$$
\mathcal{T}(t)\left(h_{n}\right)_{n \geq 0}=\left(h_{0}, T(t) h_{1}, T(t) h_{2}, \ldots\right)
$$

defines a strongly continuous semigroup of operators in $L_{\omega}$. The operator norm of $\mathcal{T}(t)$ does not exceed $e^{\omega t}$. The domain $\mathcal{D}(\mathcal{G})$ of the infinitesimal generator $\mathcal{G}$ of $\{\mathcal{T}(t), t \geq 0\}$ is composed of $\left(h_{n}\right)_{n \geq 0}$ such that $h_{n}, n \geq 1$ are absolutely continuous with $h_{n}^{\prime} \in L_{\omega}^{1}(\mathbb{R})$ and $\left(0, h_{1}^{\prime}, h_{2}^{\prime}, \ldots\right) \in L_{\omega}$. For such $\left(h_{n}\right)_{n \geq 0}$,

$$
\mathcal{G}\left(h_{n}\right)_{n \geq 0}=-\left(0, h_{1}^{\prime}, h_{2}^{\prime}, \ldots\right)
$$




\section{Constructing the Images}

As explained in the introduction to [20], given an $\left(f_{m}\right)_{m \geq 0} \in \mathcal{D}\left(B_{\mu, \alpha}\right)$ it is a good idea to look for the sequence $\left(f_{m}^{\diamond}\right)_{m \geq 0} \in L_{\omega}$ of extensions of its terms satisfying

$$
F_{n} \mathcal{T}(t)\left(f_{m}^{\diamond}\right)_{m \geq 0}=0, \quad \text { for all } n \geq 1, t \geq 0,
$$

where $F_{n}$ given by (8) is now treated as a functional defined on $\mathcal{D}(\mathcal{G})$. This condition means that

$$
f_{1}^{\diamond}(-t)=\int_{0}^{\infty} \mu(x) f_{2}^{\diamond}(x-t) \mathrm{d} x+\alpha f_{0}
$$

and

$$
f_{n}^{\diamond}(-t)=\int_{0}^{\infty} \mu(x) f_{n+1}^{\diamond}(x-t) \mathrm{d} x, \quad \text { for all } n \geq 2, t \geq 0,
$$

and thus may be rewritten as a requirement for

$$
g_{n}(x):=f_{n}^{\diamond}(-x), \quad x \geq 0
$$

as follows:

$$
\begin{aligned}
g_{1}(x) & =\int_{0}^{x} g_{2}(x-y) \mu(y) \mathrm{d} y+\int_{0}^{\infty} \mu(x+y) f_{2}(y) \mathrm{d} y+\alpha f_{0}, \\
g_{n}(x)= & \int_{0}^{x} g_{n+1}(x-y) \mu(y) \mathrm{d} y+\int_{0}^{\infty} \mu(x+y) f_{n+1}(y) \mathrm{d} y, \quad n \geq 2, x \geq 0 .
\end{aligned}
$$

This in turn simply means that we need to have

$$
g_{1}=\mu * g_{2}+U f_{2}+\alpha f_{0}, \quad g_{n}=\mu * g_{n+1}+U f_{n+1}, \quad n \geq 2,
$$

where $U f(x)=\int_{0}^{\infty} \mu(x+y) f(y) \mathrm{d} y, f \in L^{1}\left(\mathbb{R}^{+}\right)$.

We note that while condition (12) may be formulated only for $\left(f_{n}\right)_{n \geq 0}$ in $\mathcal{D}\left(B_{\mu, \alpha}\right),(13)$ makes sense for all $\left(f_{n}\right)_{n \geq 0}$ in $L$.

Lemma 1. Fix $\omega>\|\mu\|_{\infty}:=\sup _{x \geq 0} \mu(x)$. For each $\left(f_{n}\right)_{n \geq 0} \in L$ there is precisely one $\left(g_{n}\right)_{n \geq 1}$ such that (13) holds and the sequence $\left(f_{n}^{\diamond}\right)_{n \geq 0}$ defined by

$$
\begin{aligned}
f_{0}^{\diamond} & =f_{0}, \\
f_{n}^{\diamond}(x) & =f_{n}(x), \quad x \geq 0, \\
f_{n}^{\diamond}(x) & =g_{n}(-x), \quad x<0,
\end{aligned}
$$

is a member of $L_{\omega}$.

Proof. and

1. Let $K_{\omega}:=l^{1}\left(L_{\omega}^{1}\left(\mathbb{R}^{+}\right)\right)$be the space of sequences $\left(k_{n}\right)_{n \geq 1}$ such that $k_{n} \in L_{\omega}^{1}\left(\mathbb{R}^{+}\right)$

$$
\left\|\left(k_{n}\right)_{n \geq 1}\right\|_{K_{\omega}}:=\sum_{n=1}^{\infty}\left\|k_{n}\right\|_{L_{\omega}^{1}\left(\mathbb{R}^{+}\right)}<\infty
$$


Our task is thus to show that for any $\left(f_{n}\right)_{n \geq 0} \in L$, there is precisely one $\left(g_{n}\right)_{n \geq 1} \in K_{\omega}$ satisfying (13).

2. We start the proof by noting that $U f$ defined below (13) belongs to $L_{\omega}^{1}\left(\mathbb{R}^{+}\right)$and

$$
\|U f\|_{L_{\omega}^{1}\left(\mathbb{R}^{+}\right)} \leq \frac{\|\mu\|_{\infty}}{\omega}\|f\|_{L^{1}\left(\mathbb{R}^{+}\right)} .
$$

This is showed by the following calculation:

$$
\begin{aligned}
\|U f\|_{L_{\omega}^{1}\left(\mathbb{R}^{+}\right)} & \leq \int_{0}^{\infty} \mathrm{e}^{-\omega x} \int_{0}^{\infty} \mu(x+y)|f(y)| \mathrm{d} y \mathrm{~d} x \leq \\
& \leq \int_{0}^{\infty} \mathrm{e}^{-\omega x}\|\mu\|_{\infty} \int_{0}^{\infty}|f(y)| \mathrm{d} y \mathrm{~d} x=\frac{\|\mu\|_{\infty}}{\omega}\|f\|_{L^{1}\left(\mathbb{R}^{+}\right)} .
\end{aligned}
$$

Inequality (14) implies that

$$
\mathcal{U}\left(f_{n}\right)_{n \geq 0}:=\left(U f_{2}+\alpha f_{0}, U f_{3}, U f_{4}, \ldots\right)
$$

is a member of $K_{\omega}$ with

$$
\left\|\mathcal{U}\left(f_{n}\right)_{n \geq 0}\right\|_{K_{\omega}} \leq \sum_{n=2}^{\infty}\left\|U f_{n}\right\|_{L_{\omega}^{1}\left(\mathbb{R}^{+}\right)}+\frac{\alpha\left|f_{0}\right|}{\omega} \leq \frac{\max \left(\|\mu\|_{\infty}, \alpha\right)}{\omega}\left\|\left(f_{n}\right)_{n \geq 0}\right\|_{L} .
$$

3. Finally, for a fixed $\left(f_{n}\right)_{n \geq 0} \in L$, let $\mathcal{M}: K_{\omega} \rightarrow K_{\omega}$ be the map given by

$$
\mathcal{M}\left(k_{n}\right)_{n \geq 1}=\left(\mu * k_{n+1}\right)_{n \geq 1}+\mathcal{U}\left(f_{n}\right)_{n \geq 0}
$$

For any $k \in L_{\omega}^{1}\left(\mathbb{R}^{+}\right)$,

$$
\|\mu * k\|_{L_{\omega}^{1}\left(\mathbb{R}^{+}\right)} \leq\|\mu\|_{L_{\omega}^{1}\left(\mathbb{R}^{+}\right)}\|k\|_{L_{\omega}^{1}\left(\mathbb{R}^{+}\right)} \leq \frac{\|\mu\|_{\infty}}{\omega}\|k\|_{L_{\omega}^{1}\left(\mathbb{R}^{+}\right)} .
$$

It follows that

$$
\left\|\left(\mu * k_{n+1}\right)_{n \geq 1}\right\|_{K_{\omega}} \leq \frac{\|\mu\|_{\infty}}{\omega}\left\|\left(k_{n}\right)_{n \geq 1}\right\|_{K_{\omega}}
$$

Since $\|\mu\|_{\infty}<\omega, \mathcal{M}$ is a contraction mapping and Banach's fixed point theorem implies that there is precisely one $\left(g_{n}\right)_{n \geq 1} \in K_{\omega}$ such that $\mathcal{M}\left(g_{n}\right)_{n \geq 1}=\left(g_{n}\right)_{n \geq 1}$, i.e. precisely one $\left(g_{n}\right)_{n \geq 1} \in K_{\omega}$ satisfying (13).

The sequence $\left(g_{n}\right)_{n \geq 1}$, whose existence has just been established, will be called the $(\mu, \alpha)$-image of $\left(f_{n}\right)_{n \geq 0}$, and $\left(f_{n}^{\diamond}\right)_{n \geq 0}$ will be called the $(\mu, \alpha)$-extension of $\left(f_{n}\right)_{n \geq 0}$. We note that for non-negative $\left(f_{n}\right)_{n \geq 0}$, the vector $\mathcal{U}\left(f_{n}\right)_{n \geq 0}$ is also non-negative, and thus the map $\mathcal{M}$ defined in (16) leaves the non-negative cone in $K_{\omega}$ invariant. Since the fix-point of $\mathcal{M}$ may be obtained as $\lim _{l \rightarrow \infty} \mathcal{M}^{l}\left(k_{n}\right)_{n \geq 1}$ for any $\left(k_{n}\right)_{n \geq 1} \in K_{\omega}$ and in particular we may take $\left(k_{n}\right)_{n \geq 1}=\mathcal{U}\left(f_{n}\right)_{n \geq 0}$ it follows that $\left(g_{n}\right)_{n \geq 1}$ is non-negative provided $\left(f_{n}\right)_{n \geq 0}$ is.

It is worth noting that (18) forces

$$
\left\|\mathcal{M}\left(k_{n}\right)_{n \geq 1}\right\|_{K_{\omega}} \leq \frac{\|\mu\|_{\infty}}{\omega}\left\|\left(k_{n}\right)_{n \geq 1}\right\|_{K_{\omega}}+\left\|\mathcal{U}\left(f_{n}\right)_{n \geq 0}\right\|_{K_{\omega}} .
$$


Thus, by induction,

$$
\left\|\mathcal{M}^{\ell} \mathcal{U}\left(f_{n}\right)_{n \geq 0}\right\|_{K_{\omega}} \leq \sum_{i=0}^{\ell}\left(\frac{\|\mu\|_{\infty}}{\omega}\right)^{i}\left\|\mathcal{U}\left(f_{n}\right)_{n \geq 0}\right\|_{K_{\omega}}, \quad \ell \geq 1
$$

and so, using (15) and $\left(g_{n}\right)_{n \geq 1}=\lim _{\ell \rightarrow \infty} \mathcal{M}^{\ell} \mathcal{U}\left(f_{n}\right)_{n \geq 0}$,

$$
\left\|\left(g_{n}\right)_{n \geq 1}\right\|_{K_{\omega}} \leq \frac{\omega}{\omega-\|\mu\|_{\infty}}\left\|\mathcal{U}\left(f_{n}\right)_{n \geq 0}\right\|_{K_{\omega}} \leq \frac{\max \left(\|\mu\|_{\infty}, \alpha\right)}{\omega-\|\mu\|_{\infty}}\left\|\left(f_{n}\right)_{n \geq 0}\right\|_{L} .
$$

We also need information on regularity of $(\mu, \alpha)$-images, contained in Lemma 3 (see later on) which in turn is based on the following Sobolev type inequality.

Lemma 2. Let $\omega \geq 0$. Suppose $f \in L_{\omega}^{1}\left(\mathbb{R}^{+}\right)$is absolutely continuous with $f^{\prime} \in L_{\omega}^{1}\left(\mathbb{R}^{+}\right)$. Then

$$
|f(0)| \leq \omega\|f\|_{L_{\omega}^{1}\left(\mathbb{R}^{+}\right)}+\left\|f^{\prime}\right\|_{L_{\omega}^{1}\left(\mathbb{R}^{+}\right)} .
$$

Proof. The function $x \mapsto \mathrm{e}^{-\omega x} f(x)$ is also absolutely continuous with derivative equal to $\mathrm{e}^{-\omega x} f^{\prime}(x)-\omega \mathrm{e}^{-\omega x} f(x)$. For $x \geq 0$ we have thus

$$
\mathrm{e}^{-\omega x} f(x)-f(0)=\int_{0}^{x}\left[\mathrm{e}^{-\omega y} f(y)\right]^{\prime} \mathrm{d} y
$$

Since the limit as $x \rightarrow \infty$ of the right-hand side exists, so must the $\operatorname{limit}_{\lim } \rightarrow \infty \mathrm{e}^{-\omega x} f(x)$. But the latter must be zero, $x \mapsto \mathrm{e}^{-\omega x} f(x)$ being integrable. Therefore,

$$
|f(0)|=\left|\int_{0}^{\infty}\left[\omega \mathrm{e}^{-\omega y} f(y)-\mathrm{e}^{-\omega y} f^{\prime}(y)\right] \mathrm{d} y\right| \leq \omega\|f\|_{L_{\omega}^{1}\left(\mathbb{R}^{+}\right)}+\left\|f^{\prime}\right\|_{L_{\omega}^{1}\left(\mathbb{R}^{+}\right)},
$$

as desired.

Lemma 3. Fix $\left(f_{n}\right)_{n \geq 1} \in \mathcal{D}$ (see Section 1). Then $g_{n}, n \geq 1$ are absolutely continuous with $g_{n}^{\prime} \in L_{\omega}^{1}\left(\mathbb{R}^{+}\right)$and

$$
\left\|\left(g_{n}^{\prime}\right)_{n \geq 1}\right\|_{K_{\omega}}=\sum_{n=1}^{\infty}\left\|g_{n}^{\prime}\right\|_{L_{\omega}^{1}\left(\mathbb{R}^{+}\right)}<\infty
$$

Proof.

1. Suppose $f \in L^{1}\left(\mathbb{R}^{+}\right)$is absolutely continuous with $f^{\prime} \in L^{1}\left(\mathbb{R}^{+}\right)$. Then $U f$ (defined right after (13)) is absolutely continuous also, and

$$
-(U f)^{\prime}=U f^{\prime}+f(0) \mu .
$$

2. Let $\left(k_{n}\right)_{n \geq 1}:=\mathcal{U}\left(f_{n}\right)_{n \geq 0} \in K_{\omega}$. By point 1 , each $k_{n}$ is absolutely continuous with $-k_{n}^{\prime}=U f_{n+1}^{\prime}+f_{n+1}(0) \mu$. Therefore, by Lemma 2 with $\omega=0$ and (14),

$$
\begin{aligned}
\sum_{n=1}^{\infty}\left\|k_{n}^{\prime}\right\|_{L_{\omega}^{1}\left(\mathbb{R}^{+}\right)} & \leq \sum_{n=2}^{\infty}\left\|U f_{n}^{\prime}\right\|_{L_{\omega}^{1}\left(\mathbb{R}^{+}\right)}+\|\mu\|_{L_{\omega}^{1}\left(\mathbb{R}^{+}\right)} \sum_{n=2}^{\infty}\left|f_{n}(0)\right| \leq \\
& \leq \frac{\|\mu\|_{\infty}}{\omega} \sum_{n=2}^{\infty}\left\|f_{n}^{\prime}\right\|_{L^{1}\left(\mathbb{R}^{+}\right)}+\frac{\|\mu\|_{\infty}}{\omega} \sum_{n=2}^{\infty}\left\|f_{n}^{\prime}\right\|_{L^{1}\left(\mathbb{R}^{+}\right)}<\infty .
\end{aligned}
$$


Combining this with (15) we see that

$$
\left\|\left(k_{n}\right)_{n \geq 1}\right\|_{K_{\omega}}+\left\|\left(k_{n}^{\prime}\right)_{n \geq 1}\right\|_{K_{\omega}}<\infty
$$

3. An induction argument shows that the $n$-th coordinate of $\mathcal{M}^{\ell}\left(k_{n}\right)_{n \geq 1}$ is $\sum_{i=0}^{\ell} \mu^{i *} *$ $k_{i+n}, \ell \geq 1$. Since $\left(g_{n}\right)_{n \geq 1}=\lim _{\ell \rightarrow \infty} \mathcal{M}^{\ell}\left(k_{n}\right)_{n \geq 1}$ we have

$$
g_{n}=\sum_{i=0}^{\infty} \mu^{i *} * k_{i+n}, \quad n \geq 1 .
$$

4. If a $k \in L_{\omega}^{1}\left(\mathbb{R}^{+}\right)$is absolutely continuous with $k^{\prime} \in L_{\omega}^{1}\left(\mathbb{R}^{+}\right)$, then so is $\mu * k$ and $(\mu * k)^{\prime}=\mu * k^{\prime}+k(0) \mu$. Since $\left(\mu^{i *} * k\right)(0)=0$ for $i \geq 1$, an induction argument shows that

$$
\left(\mu^{i *} * k\right)^{\prime}=\mu^{i *} * k^{\prime}+k(0) \mu^{i *}, \quad i \geq 1
$$

This in turn yields

$$
\left(\sum_{i=0}^{\ell} \mu^{i *} * k_{i+n}\right)^{\prime}=\sum_{i=0}^{\ell} \mu^{i *} * k_{i+n}^{\prime}+\sum_{i=1}^{\ell} k_{i+n}(0) \mu^{i *}
$$

5. We claim that

$$
\sum_{n=1}^{\infty}\left(\sum_{i=0}^{\infty}\left\|\mu^{i *} * k_{i+n}^{\prime}\right\|_{L_{\omega}^{1}\left(\mathbb{R}^{+}\right)}+\sum_{i=1}^{\infty}\left\|k_{i+n}(0) \mu^{i *}\right\|_{L_{\omega}^{1}\left(\mathbb{R}^{+}\right)}\right)<\infty .
$$

Since (use (17))

$$
\begin{aligned}
\sum_{n=1}^{\infty} \sum_{i=0}^{\infty}\left\|\mu^{i *} * k_{i+n}^{\prime}\right\|_{L_{\omega}^{1}\left(\mathbb{R}^{+}\right)} & \leq \sum_{n=1}^{\infty} \sum_{i=0}^{\infty}\left(\frac{\|\mu\|_{\infty}}{\omega}\right)^{i}\left\|k_{i+n}^{\prime}\right\|_{L_{\omega}^{1}\left(\mathbb{R}^{+}\right)} \\
& \leq \sum_{i=0}^{\infty}\left(\frac{\|\mu\|_{\infty}}{\omega}\right)^{i} \sum_{n=1}^{\infty}\left\|k_{n}^{\prime}\right\|_{L_{\omega}^{1}\left(\mathbb{R}^{+}\right)} \\
& =\frac{\omega}{\omega-\|\mu\|_{\infty}}\left\|\left(k_{n}^{\prime}\right)_{n \geq 1}\right\|_{K_{\omega}}
\end{aligned}
$$

is finite by (20), to prove (22) we need to estimate

$$
\sum_{n=1}^{\infty} \sum_{i=1}^{\infty}\left\|k_{i+n}(0) \mu^{i *}\right\|_{L_{\omega}^{1}\left(\mathbb{R}^{+}\right)}
$$

By Lemma 2, however, this quantity does not exceed

$$
\begin{aligned}
& \sum_{i=1}^{\infty}\left(\frac{\|\mu\|_{\infty}}{\omega}\right)^{i} \sum_{n=1}^{\infty}\left[\omega\left\|k_{n}\right\|_{L_{\omega}^{1}\left(\mathbb{R}^{+}\right)}+\left\|k_{n}^{\prime}\right\|_{L_{\omega}^{1}\left(\mathbb{R}^{+}\right)}\right]= \\
& =\frac{\|\mu\|_{\infty}}{\omega-\|\mu\|_{\infty}}\left(\omega\left\|\left(k_{n}\right)_{n \geq 1}\right\|_{K_{\omega}}+\left\|\left(k_{n}^{\prime}\right)_{n \geq 1}\right\|_{K_{\omega}}\right)
\end{aligned}
$$

and this is finite by (20). This completes the proof of (22). 
6. Let $\mathcal{D}(D) \subset L_{\omega}^{1}\left(\mathbb{R}^{+}\right)$be the set of absolutely continuous $f \in L_{\omega}^{1}\left(\mathbb{R}^{+}\right)$such that $f^{\prime} \in L_{\omega}^{1}\left(\mathbb{R}^{+}\right)$, and let $D f=f^{\prime}$. A straightforward argument shows that $D$ is closed. Inequality (22) implies that for $n \geq 1$, the series

$$
k_{n}^{\prime}+\sum_{i=1}^{\infty}\left[\mu^{i *} * k_{i+n}^{\prime}+k_{i+n}(0) \mu^{i *}\right]
$$

converges absolutely in $L_{\omega}^{1}\left(\mathbb{R}^{+}\right)$. It follows that $D\left(\sum_{i=0}^{\ell} \mu^{i *} * k_{i+n}\right)$ (calculated in point 4.) converges to the sum of this series. Since $D$ is closed, $g_{n}$ (given by $(21)$ ) belongs to $\mathcal{D}(D)$, i.e., it is absolutely continuous with $g_{n}^{\prime}$ equal to the sum of the series (23). Combining

$$
\left\|g_{n}^{\prime}\right\|_{L_{\omega}^{1}\left(\mathbb{R}^{+}\right)} \leq \sum_{i=0}^{\infty}\left\|\mu^{i *} * k_{i+n}^{\prime}\right\|_{L_{\omega}^{1}\left(\mathbb{R}^{+}\right)}+\sum_{i=1}^{\infty}\left\|k_{i+n}(0) \mu^{i *}\right\|_{L_{\omega}^{1}\left(\mathbb{R}^{+}\right)}
$$

and (22), we complete the proof.

\section{Abstract Kelvin Formula for $\left\{\mathrm{e}^{t B_{\mu, \alpha}}, t \geq 0\right\}$}

The stage is now ready for the proof of Theorem 2. In fact, we will show the following result, giving a somewhat deeper insight into the nature of the semigroup generated by $B_{\mu, \alpha}$.

Theorem 3. Let $R: L_{\omega}^{1}(\mathbb{R}) \rightarrow L^{1}\left(\mathbb{R}^{+}\right)$map an $f \in L_{\omega}^{1}(\mathbb{R})$ to its restriction $f_{\mid[0, \infty)}$, and let $\mathcal{R}: L_{\omega} \rightarrow L$ be defined by

$$
\mathcal{R}\left(f_{n}\right)_{n \geq 0}=\left(f_{0}, R f_{1}, R f_{2}, R f_{3}, \ldots\right) .
$$

Also, let $\mathcal{E}: L \rightarrow L_{\omega}$ map elements of $L$ to their $(\mu, \alpha)$-extensions. The abstract Kelvin formula

$$
\mathcal{S}(t)=\mathcal{R} \mathcal{T}(t) \mathcal{E}, \quad t \geq 0
$$

where $\{\mathcal{T}(t), t \geq 0\}$ is the translation semigroup of (11), defines a strongly continuous semigroup of operators in $L$, and the infinitesimal generator of $\{\mathcal{S}(t), t \geq 0\}$ is $B_{\mu, \alpha}$.

To see that Theorem 2 is a direct consequence of Theorem 3 it suffices to recall from the previous section that $(\mu, \alpha)$-extensions of non-negative $\left(f_{n}\right)_{n \geq 0}$ are non-negative, and that $\{\mathcal{T}(t), t \geq 0\}$ is a semigroup of non-negative operators.

The proof of Theorem 3 will become more clear if we extract from it the following lemma.

Lemma 4. Let $\left(f_{n}\right)_{n \geq 0} \in \mathcal{D}\left(B_{\mu, \alpha}\right)$ be fixed.

(a) $\left(f_{n}^{\diamond}\right)_{n \geq 0}$, the $(\mu, \alpha)$-extension of $\left(f_{n}\right)_{n \geq 0}$, belongs to the domain $\mathcal{D}(\mathcal{G})$ of the infinitesimal generator of the translation semigroup (11),

(b) For all $t \geq 0, \mathcal{T}(t)\left(f_{n}\right)_{n \geq 0}$ is the $(\mu, \alpha)$-extension of its own restriction $\mathcal{R} \mathcal{T}(t)\left(f_{n}\right)_{n \geq 0}$. 
Proof. (a) By Lemma 3, our assumption implies that each $g_{n}, n \geq 1$ is absolutely continuous with $g_{n}^{\prime} \in L_{\omega}^{1}\left(\mathbb{R}^{+}\right)$. Moreover, by definition of $g_{n}$,

$$
\begin{aligned}
& g_{1}(0)=U f_{2}(0)+\alpha f_{0}=\int_{0}^{\infty} \mu(y) f_{2}(y) \mathrm{d} y+\alpha f_{0} \\
& g_{n}(0)=U f_{n+1}(0)=\int_{0}^{\infty} \mu(y) f_{n+1}(y) \mathrm{d} y, \quad n \geq 2 .
\end{aligned}
$$

Since $\left(f_{m}\right)_{m \geq 0} \in \bigcap_{n \geq 1} \operatorname{ker} F_{n}, f_{n}(0)=g_{n}(0)$ and so $f_{n}^{\diamond}$ is absolutely continuous for all $n \geq 1$. Moreover, by

$$
\left\|\left(f_{n}^{\diamond}\right)^{\prime}\right\|_{L_{\omega}^{1}(\mathbb{R})}=\left\|f_{n}^{\prime}\right\|_{L^{1}\left(\mathbb{R}^{+}\right)}+\left\|g_{n}^{\prime}\right\|_{L_{\omega}^{1}\left(\mathbb{R}^{+}\right)}
$$

Lemma 3 implies

$$
\sum_{n=1}^{\infty}\left\|\left(f_{n}^{\diamond}\right)^{\prime}\right\|_{L_{\omega}^{1}(\mathbb{R})}<\infty
$$

completing the proof of $(a)$.

(b) Fix $t \geq 0$. By $(a),\left(f_{n}^{\diamond}\right)_{n \geq 0}$ is a member of $\mathcal{D}(\mathcal{G})$. It follows that so is $\left(f_{n}^{\boldsymbol{\alpha}}\right)_{n \geq 0}:=$ $\mathcal{T}(t)\left(f_{n}^{\diamond}\right)_{n \geq 0}$ and that $F_{n} \mathcal{T}(s)\left(f_{m}^{\diamond}\right)_{m \geq 0}=0$ for all $s \geq 0$ and $n \geq 1$ (on $\mathcal{D}(\mathcal{G}),(12)$ and (13) are equivalent). Therefore, for all $s$ and $n \geq 1, F_{n} \mathcal{T}(s)\left(f_{m}^{\mathbf{p}}\right)_{m \geq 0}=F_{n} \mathcal{T}(s+t)\left(f_{m}^{\diamond}\right)_{m \geq 0}=0$. This means, by definition, that $\left(f_{m}^{\boldsymbol{m}}\right)_{m \geq 0}$ is the $(\mu, \alpha)$-extension (of its own restriction).

\section{Proof of Theorem 3}

1. Fix $\omega>\|\mu\|_{\infty}$, and let $E_{\omega} \subset L_{\omega}$ be the space of $(\mu, \alpha)$-extensions of members of $L$. Inequality (19) shows that $\mathcal{E}$ mapping $L$ onto $E_{\omega}$ is bounded. Since $\mathcal{E}$ has a bounded inverse $\mathcal{R}, E_{\omega}$ is closed in $L_{\omega}$, and hence is a Banach space (with norm inherited from $L_{\omega}$ ). The spaces $L$ and $E_{\omega}$ are isomorphic with the isomorphism $\mathcal{E}: L \rightarrow E_{\omega}$ and its inverse $\mathcal{R}: E_{\omega} \rightarrow L$.

2. Since $\mathcal{D}\left(B_{\mu, \alpha}\right)$ is dense in $L$ (as a straightforward argument shows), so is its image $\mathcal{E D}\left(B_{\mu, \alpha}\right)$ in $E_{\omega}$. Lemma 4 now says that $\mathcal{E D}\left(B_{\mu, \alpha}\right)$ is invariant for the translation semigroup $\{\mathcal{T}(t), t \geq 0\}$. It follows that so is $E_{\omega}$. Hence, $\{\mathcal{T}(t), t \geq 0\}$ restricted to $E_{\omega}$ is a strongly continuous semigroup. The semigroup defined by the abstract Kelvin formula (24) is thus the isomorphic image of $\{\mathcal{T}(t), t \geq 0\}$ restricted to $E_{\omega}$, and it is obviously strongly continuous.

3. We are left with showing that the generator of $\{\mathcal{S}(t), t \geq 0\}$ is $B_{\mu, \alpha}$. To this end, we recall that the generator of $\{\mathcal{T}(t), t \geq 0\}$ restricted to $E_{\omega}$ is the part $\mathcal{G}_{\mathrm{p}}$ of $\mathcal{G}$ in $E_{\omega}(\mathcal{G}$ was defined in Section 3). Thus $\left(f_{n}^{\diamond}\right)_{n \geq 0} \in E_{\omega}$ is a member of $\mathcal{D}\left(\mathcal{G}_{\mathrm{p}}\right)\left(=\mathcal{D}(\mathcal{G}) \cap E_{\omega}\right)$ iff $f_{n}^{\diamond}, n \geq 1$ are absolutely continuous and $\left(0,\left(f_{1}^{\diamond}\right)^{\prime},\left(f_{2}^{\diamond}\right)^{\prime}, \ldots\right) \in L_{\omega}$; then

$$
\mathcal{G}_{\mathrm{p}}\left(f_{n}^{\diamond}\right)_{n \geq 0}=-\left(0,\left(f_{1}^{\diamond}\right)^{\prime},\left(f_{2}^{\diamond}\right)^{\prime}, \ldots\right)
$$

the vector on the right-hand side here automatically belongs to $E_{\omega}$ since $E_{\omega}$ is invariant for the translation semigroup.

On the other hand, $\left(f_{n}\right)_{n \geq 0}$ belongs to the domain of the generator, say $\mathcal{G}_{1}$, of $\{\mathcal{S}(t), t \geq$ $0\}$ iff $\left(f_{n}^{\diamond}\right)_{n \geq 0}=\mathcal{E}\left(f_{n}\right)_{n \geq 0}$ belongs to $\mathcal{D}\left(\mathcal{G}_{\mathrm{p}}\right)$. Lemma 4 tells us that for $\left(f_{n}\right)_{n \geq 0} \in \mathcal{D}\left(B_{\mu, \alpha}\right)$ 
the latter condition holds. Conversely, if $\left(f_{n}^{\diamond}\right)_{n \geq 0}$ is a member of $\mathcal{D}(\mathcal{G}) \cap E_{\omega}$, then each $f_{n}$ (being the restriction of $f_{n}^{\diamond}$ ) must be absolutely continuous with $f_{n}^{\prime} \in L^{1}\left(\mathbb{R}^{+}\right)$, and we must have

$$
\sum_{n=1}^{\infty}\left\|f_{n}^{\prime}\right\|_{L^{1}\left(\mathbb{R}^{+}\right)} \leq \sum_{n=1}^{\infty}\left\|\left(f_{n}^{\diamond}\right)^{\prime}\right\|_{L_{\omega}^{1}(\mathbb{R})}<\infty .
$$

Also, absolute continuity of $f_{n}^{\diamond}$ implies $f_{n}(0)=g_{n}(0)$ for all $n$, and then a look at $(25)$ reveals that $\left(f_{n}\right)_{n \geq 0} \in \bigcap_{n \geq 1}$ ker $F_{n}$, thus showing that $\mathcal{D}\left(\mathcal{G}_{1}\right)=\mathcal{D}\left(B_{\mu, \alpha}\right)$.

For such $\left(f_{n}\right)_{n \geq 0}$,

$$
\begin{aligned}
\mathcal{G}_{1}\left(f_{n}\right)_{n \geq 0} & =\mathcal{R G} \mathcal{E}\left(f_{n}\right)_{n \geq 0}=\mathcal{R G}\left(f_{n}^{\diamond}\right)_{n \geq 0}= \\
& =-\mathcal{R}\left(0,\left(f_{1}^{\diamond}\right)^{\prime},\left(f_{2}^{\diamond}\right)^{\prime}, \ldots\right)=-\left(0, f_{1}^{\prime}, f_{2}^{\prime}, \ldots\right)=B_{\mu, \alpha}\left(f_{n}\right)_{n \geq 0}
\end{aligned}
$$

This completes the proof.

Acknowledgment. I would like to thank Lukasz Stepien for careful reading of the manuscript and for many remarks that helped to improve the presentation of the material discussed here.

\section{References}

1. Engel K.-J., Nagel R. One-Parameter Semigroups for Linear Evolution Equations. N.Y., Springer, 2000.

2. Bátkai A., Piazzera S. Semigroups for Delay Equations. Wellesley, CRC Press, 2005.

3. Siegle P., Goychuk I., Talkner P., Hänggi P. Markovian Embedding of Non-Markovian Superdiffusion. Physical Review, 2010, vol. 81, Article ID 011136, 10 p.

4. Durbin R., Eddy S., Krogh A., Mitchison G. Biological Sequence Analysis. Probabilistic Models of Proteins and Nucleic Acids. Cambridge, Cambridge University Press, 1998.

5. Hoek J., Elliott R.J. Introduction to Hidden Semi-Markov Models. Cambridge, Cambridge University Press, 2018.

6. Goldstein S. On Diffusion by Discontinuous Movements, and on the Telegraph Equation. The Quarterly Journal of Mechanics and Applied Mathematics, 1986, vol. 4, no. 2, pp. 129-156.

7. Kac M. Some Stochastic Problems in Physics and Mechanics. N.Y., Literary Licensing, 1956.

8. Kisyński J. On M. Kac's Probabilistic Formula for the Solutions of the Telegraphist's Equation. Annales Polonici Mathematici, 1974, vol. 29, pp. 259-272.

9. Ethier S.N., Kurtz, T.G. Markov Processes. Characterization and Convergence. N.Y., Wiley, 1986 .

10. Pinsky M.A. Lectures on Random Evolutions. Singapore, World Scientific, 1991.

11. Asmussen S. Applied Probability and Queues. N.Y., Springer, 2003.

12. Cox D.R. The Analysis of Non-Markovian Stochastic Processes by the Inclusion of Supplementary Variables. Mathematical Proceedings of the Cambridge Philosophical Society, 1955, vol. 51, no. 3, pp. 433-441.

13. Gwiżdż P. Application of Stochastic Semigroups to Queueing Models. Annales Mathematicae Silesianae, 2018 (to appear). DOI: 10.2478/amsil-2018-0007

14. Davis M.H.A. Lectures on Stochastic Control and Nonlinear Filtering. N.Y., Springer, 1984. 
15. Davis M.H.A. Piece-Wise Deterministic Markov Processes. Journal of the Royal Statistical Society, 1984, vol. 46, no. 3, pp. 353-388.

16. Davis M.H.A. Markov Processes and Optimization. London, Chapman and Hall, 1993.

17. Rudnicki R., Tyran-Kamińska M. Piecewise Deterministic Processes in Biological Models. N.Y., Springer, 2017.

18. Greiner G. Perturbing the Boundary Conditions of a Generator. Houston Journal of Mathematics, 1987, vol. 13, no. 2, pp. 213-229.

19. Bobrowski A. Generation of Cosine Families via Lord Kelvin's Method of Images. Journal of Evolution Equations, 2010, vol. 10, no. 3, pp. 663-675.

20. Bobrowski A. Lord Kelvin's Method of Images in the Semigroup Theory. Semigroup Forum, 2010, vol. 81, no. 3, pp. 435-445.

21. Bobrowski A. Families of Operators Describing Diffusion Through Permeable Membranes. Operator Semigroups Meet Complex Analysis, Harmonic Analysis and Mathematical Physics, 2015, vol. 250, pp. $87-105$.

22. Bobrowski A., Gregosiewicz A. A General Theorem on Generation of Moments-Preserving Cosine Families by Laplace Operators in C[0,1]. Semigroup Forum, 2014, vol. 88, no. 3, pp. 689-701.

23. Bobrowski A., Gregosiewicz A., Murat M. Functionals-Preserving Cosine Families Generated by Laplace Operators in C[0,1]. Discrete and Continuous Dynamical System, 2015, vol. 20, no. 7 , pp. $1877-1895$.

24. Bobrowski A., Mugnolo D. On Moments-Preserving Cosine Families and Semigroups in $C[0,1]$. Journal of Evolution Equations, 2013, vol. 13, no. 4, pp. 715-735.

25. Bobrowski A. From Diffusions on Graphs to Markov Chains via Asymptotic State Lumping. Annales Henri Poincare, 2012, vol. 13, no. 6, pp. 1501-1510.

26. Bobrowski A., Kaźmierczak B., Kunze M. An Averaging Principle for Fast Diffusions in Domains Separated by Semi-Permeable Membranes. Mathematical Models and Methods in Applied Sciences, 2017, vol. 27, no. 4, pp. 663-706.

Received June 21, 2018

УДК $517.9+519.21+517.958$

DOI: $10.14529 / \mathbf{m m p} 180303$

\section{ЛОРД КЕЛЬВИН И АНДРЕЙ АНДРЕЕВИЧ МАРКОВ К ОЧЕРЕДИ С ОДИНОЧНОГО СЕРВЕРА}

$\boldsymbol{A}$. Бобровский, Люблинский технологический университет, г. Люблин, Польша

Мы используем метод изображений лорда Кельвина, чтобы показать, что некоторая бесконечная система уравнений с интересными граничными условиями приводит к марковской динамике в пространстве $L^{1}$-типа. Эта система берет свое начало в теории массового обслуживания.

Ключевые слова: очередъ; метод изображений; теорема генерачии; граничные условия; Марковскал динамика.

Адам Бобровский, профессор, кафедра математики, Люблинский технологический университет (г. Люблин, Польша), a.bobrowski@pollub.pl.

Поступила в редакцию 21 июня 2018 г. 